Bull. Austral. Math. Soc.

VOL. 57 (1998) [153-158]

\title{
ON GODEMENT'S CHARACTERISATION OF AMENABILITY
}

\author{
Alain Valette
}

Motivated by a question related to the construction of the Baum-Connes analytical assembly map for locally compact groups, we refine a criterion of Godement for amenability: for a unimodular group $G$, our criterion says that $G$ is amenable if and only if every compactly supported, positive-definite function has non-negative integral over $G$.

\section{Main Result}

Godement proved [7, pp.76-77] that a locally compact group $G$ is amenable if and only if, for every bounded positive-definite measure $\mu$ on $G$ one has

$$
\int_{G} d \mu(g) \geqslant 0
$$

(see also Dixmier's book [5, 18.3.6] or Pier's book [12, Theorem 8.9]; we shall follow Dixmier's notation). Our aim in this note is to sharpen Godement's criterion by proving:

THEOREM 1. Let $G$ be a locally compact group, with left Haar measure $d g$ and modular homomorphism $\Delta: G \rightarrow \mathbb{R}_{+}^{*}$. The following are equivalent:

1. $G$ is amenable;

2. for every compactly supported, positive-definite function $\phi$ on $G$, the function $\Delta^{-1 / 2} \phi$ defines a positive element in the full $C^{*}$-algebra $C^{*} G$;

3. for every compactly supported, positive-definite function $\phi$ on $G$, one has $\int_{G}\left(\Delta^{-1 / 2} \phi\right)(g) d g \geqslant 0$.

This theorem is indeed a strengthening of Godement's result, since a compactly supported function $\phi$ is positive-definite on $G$ if and only if the measure $\left(\Delta^{-1 / 2} \phi\right)(g) d g$ is positive-definite $[5,13.7 .6]$.

Our result illustrates a difference between the full and reduced $\mathrm{C}^{*}$-algebras; indeed, if $\phi$ is a compactly supported positive-definite function on an arbitrary locally compact group $\mathrm{G}$, then $\Delta^{-1 / 2} \phi$ defines a positive element in the reduced $\mathrm{C}^{*}$-algebra $C_{\tau}^{*} G[5$, 13.7.8].

We shall need the following simple lemma.

Received 20th May, 1997.

I thank M.E.B. Bekka and G. Pisier for some useful conversations. Recently, Bekka has shown us a functional analytic proof of the main result, in the spirit of $[5,18.3 .6]$.

Copyright Clearance Centre, Inc. Serial-fee code: 0004-9729/98 \$A2.00+0.00. 
LEMMA 1. Let $\pi$ be a unitary representation of a group $H$ on a Hilbert space $\mathcal{H}_{\pi}$. Let $T$ be a bounded operator on $\mathcal{H}_{\pi}$ that commutes with $\pi(H)$. For any $\xi \in \mathcal{H}_{\pi}$, the function

$$
\phi: \begin{cases}H & \rightarrow \mathbb{C} \\ h & \mapsto\|T\|^{2}\langle\xi \mid \pi(h) \xi\rangle-\langle T \xi \mid \pi(h) T \xi\rangle\end{cases}
$$

is positive-definite on $H$.

Proof: For $h_{1}, \ldots, h_{n} \in H, \lambda_{1}, \ldots, \lambda_{n} \in \mathbb{C}$ we have

$$
\sum_{i, j=1}^{n} \overline{\lambda_{i}} \lambda_{j} \phi\left(h_{i}^{-1} h_{j}\right)=\|T\|^{2}\left\|\sum_{i=1}^{n} \lambda_{i} \pi\left(h_{i}\right) \xi\right\|^{2}-\left\|T\left(\sum_{i=1}^{n} \lambda_{i} \pi\left(h_{i}\right) \xi\right)\right\|^{2} \geqslant 0 .
$$

ProOF OF THEOREM 1:

$(i) \Longrightarrow(i i)$ If $G$ is amenable, then $C_{r}^{*} G=C^{*} G$, so that the conclusion follows from one of the above remarks.

$(i i) \Longrightarrow(i i i)$ Integration over $G$ defines a character of $C^{*} G$; therefore, if $\Delta^{-1 / 2} \phi$ is a positive element in $C^{*} G$, then $\int_{G}\left(\Delta^{-1 / 2} \phi\right)(g) d g \geqslant 0$.

(iii) $\Longrightarrow(i)$ We are going to prove that the trivial representation of $G$ is weakly contained in the left regular representation $\lambda$ of $G$, that is,

$$
\left|\int_{G} f(g) d g\right| \leqslant\|\lambda(f)\|
$$

for every compactly supported, continuous function $f$ on $G$. Denote by $\rho$ the right regular representation of $G$ on $L^{2}(G)$. Fix a compactly supported, continuous function $\xi$, with non-zero integral over $G$. We apply the lemma with $\pi=\rho$ and $T=\lambda(f)$; it ensures that the function

$$
\phi: \begin{cases}G & \rightarrow \mathbb{C} \\ g & \mapsto\|\lambda(f)\|^{2}\langle\xi \mid \rho(g) \xi\rangle-\langle\lambda(f) \xi \mid \rho(g) \lambda(f) \xi\rangle\end{cases}
$$

is positive-definite on $G$. Since $f$ and $\xi$ have compact support, so has $\phi$. A simple computation shows:

$$
\Delta^{-1 / 2} \phi=\|\lambda(f)\|^{2}\left(\xi^{*} \star \xi\right)-(\lambda(f) \xi)^{*} \star \lambda(f) \xi .
$$

Since $\lambda(f) \xi=f \star \xi$, this gives:

$$
\Delta^{-1 / 2} \phi=\|\lambda(f)\|^{2}\left(\xi^{*} \star \xi\right)-\xi^{*} \star f^{*} \star f \star \xi
$$

By assumption $\int_{G}\left(\Delta^{-1 / 2} \phi\right)(g) d g \geqslant 0$, so that:

$$
0 \leqslant\left(\|\lambda(f)\|^{2}-\left|\int_{G} f(g) d g\right|^{2}\right)\left|\int_{G} \xi(g) d g\right|^{2} .
$$

Since $\xi$ has non-zero integral, the result follows. 


\section{REMARKS ON UNIMODULAR GROUPS}

Assume from now on that $G$ is unimodular - in this case our Theorem has an especially simple form. Let $\phi$ be a compactly supported, positive-definite function on $G$. By $[5,13.8 .6]$, there exists $\xi \in L^{2}(G)$ such that $\phi=\xi^{*} \star \xi$. If $\xi \in L^{1}(G) \cap L^{2}(G)$, then

$$
\int_{G} \phi(g) d g=\left|\int_{G} \xi(g) d g\right|^{2} \geqslant 0 .
$$

This means that, for a non-amenable $G$, we can find a compactly supported positivedefinite function $\phi$ on $G$ with $\int_{G} \phi(g) d g<0$, and the associated $\xi$ will be in $L^{2}(G)-$ $L^{1}(G)$.

Note that functions $\phi$ as above can be explicitly constructed for $G$ non-amenable. Indeed, since $G$ is the inductive limit of its compactly generated open subgroups, we may as well assume that $G$ is compactly generated. Let then $K$ be a symmetric (that is, $K=$ $K^{-1}$ ), open, relatively compact, generating subset of $G$; denote by $h$ the characteristic function of $K$. It follows from the characterisation of amenability in [1] and [4] that

$$
\|\lambda(h)\|<\operatorname{meas}(K) .
$$

Convolving $h$ with a non-negative, continuous function with integral 1 and small support around the origin, we get a non-negative, continuous, compactly supported $f$ with

$$
\|\lambda(f)\|<\int_{G} f(g) d g .
$$

Then, for any continuous, compactly supported function $\xi$ with non-zero integral on $G$, the positive-definite function

$$
\phi=\|\lambda(f)\|^{2}\left(\xi^{*} \star \xi\right)-\xi^{*} \star f^{*} \star f \star \xi
$$

appearing in the proof of the Theorem, has strictly negative integral on $G$.

If the group is discrete (and finitely generated), we may take $\xi=\delta_{e}$. This gives the following re-phrasing of Kesten's well-known characterisation of amenability [9].

Corollary 1. Let $\Gamma$ be a finitely generated group. Let $h$ be the characteristic function of a finite, symmetric, generating subset of $\Gamma$. The group $\Gamma$ is amenable if and only if the positive-definite function $\phi=\|\lambda(h)\|^{2} \cdot \delta_{e}-h \star h$ has non-negative sum on $\Gamma$.

\section{Relations with the Baum-Connes conjecture}

Our actual motivation for this paper was not to obtain a Godement-type criterion for amenability. Rather, it came from questions related to $\mathrm{K}$-theory of group $\mathrm{C}^{*}$-algebras, and it is only subsequently that we realised that it was connected to Godement's result.

Let us be more precise. For $G$ a locally compact group, Baum, Connes and Higson [2] give a conjectural model for the K-theory groups $K_{i}\left(C_{\tau}^{*} G\right)(i=0,1)$. Namely, they 
consider a space $\underline{E G}$ which is universal for proper $G$-actions (if $G$ has no non-trivial compact subgroup, this is the total space $E G$ of a contractible principal $G$-bundle), take the $G$-equivariant K-homology with compact support $K_{i}^{G}(\underline{E G})$, and construct an index map, or analytical assembly map

$$
\mu_{i}^{G}: K_{i}^{G}(\underline{E G}) \rightarrow K_{i}\left(C_{r}^{*} G\right)(i=0,1)
$$

which they conjecture to be an isomorphism. Here we wish to emphasise only those parts of the construction that pertain to group representation theory. So let us just say that an element of $K_{i}^{G}(\underline{E G})$ is given by a quadruple $(X, \alpha, \pi, F)$ where $X$ is a locally compact space endowed with a continuous, proper action of $G$ by homeomorphisms such that the orbit space $G \backslash X$ is compact, and $\alpha$ is a *-representation of the commutative $\mathrm{C}^{*}$ algebra $C_{0}(X)$ on some Hilbert space $\mathcal{H}$, which is covariant with respect to the unitary representation $\pi$ of $G$ on $\mathcal{H}$, meaning:

$$
\alpha(g(f))=\pi(g) \alpha(f) \pi\left(g^{-1}\right)
$$

( $f \in C_{0}(X), g \in G$ ); finally $F$ is a $G$-equivariant abstract elliptic operator on $X$, that is, $F$ is a Fredholm operator on $\mathcal{H}$ satisfying a number of compatibility conditions with $\alpha$ and $\pi$, as listed in [2, Definition 3.6]. The map $\mu_{i}^{G}$ converts this quadruple into a $\mathrm{K}$-theory element of $C_{r}^{*} G$, that is, a pair $(\mathcal{E}, \mathcal{F})$ where $\mathcal{E}$ is a left Hilbert $\mathrm{C}^{*}$-module over $C_{r}^{*} G$, and $\mathcal{F}$ is a Fredholm operator on $\mathcal{E}$ (in the sense of Hilbert $\mathrm{C}^{*}$-modules - this is Kasparov's version of $\mathrm{K}$-theory for $\mathrm{C}^{*}$-algebras, see [10]). To construct $\mathcal{E}$, one considers the subspace $\alpha\left(C_{c}(X)\right) \mathcal{H}$, and views it as a left module over the convolution algebra $C_{c}(G)$; as a consequence of the properness of the $G$-action on $X$, for any $\xi, \eta \in \alpha\left(C_{c}(X)\right) \mathcal{H}$, the function $g \mapsto\langle\xi \mid \pi(g) \eta\rangle$ has compact support on $G$. One defines then a $C_{c}(G)$-valued scalar product on $\alpha\left(C_{c}(X)\right) \mathcal{H}$ by the formula

$$
\langle\xi \mid \eta\rangle_{C^{*}}(g)=\Delta^{-1 / 2}(g)\langle\xi \mid \pi(g) \eta\rangle
$$

$(g \in G)$. (In passing, notice that the formula for this scalar product in [2, p.252] is correct only for unimodular groups, since it overlooks the factor $\Delta^{-1 / 2}$.) For the abstract reason mentioned in the beginning of this paper (namely, if $\phi$ is a compactly supported positive-definite function on $\mathrm{G}$, then $\Delta^{-1 / 2} \phi$ defines a positive element in $C_{\tau}^{*} G$ ), for any $\xi \in \alpha\left(C_{c}(X)\right) \mathcal{H}$ the scalar product $\langle\xi \mid \xi\rangle_{C^{*}}$ defines a positive element in $C_{r}^{*} G$. Therefore, we may complete $\alpha\left(C_{c}(X)\right) \mathcal{H}$ with respect to $\langle\cdot \mid \cdot\rangle_{C^{*}}$ and obtain the left Hilbert $\mathrm{C}^{*}$ module over $C_{r}^{*} G$. Then one checks as in [13, Section 2] that $F$ leaves $\alpha\left(C_{c}(X)\right) \mathcal{H}$ invariant, extends to a bounded operator $\mathcal{F}$ on $\mathcal{E}$, and that $\mathcal{F}$ is Fredholm on $\mathcal{E}$, so that $(\mathcal{E}, \mathcal{F})$ defines an element of $K_{i}\left(C_{r}^{*} G\right)$.

It has been noticed by several people (see for example, [3, p.99], [6, Section 6], [8]) that the assembly map $\mu_{i}^{G}$ factors through the K-theory of the full $\mathrm{C}^{*}$-algebra $C^{*} G$, that is, there exists a map

$$
\tilde{\mu}_{i}^{G}: K_{i}^{G}(\underline{E G}) \rightarrow K_{i}\left(C^{*} G\right)(i=0,1)
$$


such that $\mu_{i}^{G}=\lambda_{*} \circ \tilde{\mu}_{i}^{G}$. If, as above, we start with a quadruple $(X, \alpha, \pi, F) \in K_{i}^{G}(\underline{E G})$ and wish to describe its image under $\widetilde{\mu}_{i}^{G}$, as above we would like to complete $\alpha\left(C_{c}(X)\right) \mathcal{H}$ into a left Hilbert $C^{*}$-module over $C^{*} G$. For this, we need to know that, for $\xi \in$ $\alpha\left(C_{c}(X)\right) \mathcal{H}$, the scalar product $\langle\xi \mid \xi\rangle_{C^{*}}$ defines a positive element in $C^{*} G$. At this point, we want to stress the fact - never mentioned explicitly in the literature - that the abstract reason that works for $C_{r}^{*} G$ fails in general for $C^{*} G$; indeed, the main result of this note shows that this abstract argument only works for $G$ amenable.

To prove that $\langle\xi \mid \xi\rangle_{C^{*}}$ is a positive element in $C^{*} G$, we have to exploit in a deeper way the properness of the $G$-action on $X$. It follows from the slice map theorem of Palais [11] that the action of $G$ on $X$ is locally induced from actions of compact subgroups, that is, that $X$ is locally of the form $G \times{ }_{K} S$, where $K$ is a compact subgroup of $G$ and $S$ is a locally compact space with a $K$-action. On the other hand, it follows from the representation theory of abelian $\mathrm{C}^{*}$-algebras that the representation $\alpha$ of $C_{0}(X)$ is unitarily equivalent to the representation by pointwise multiplication on $L^{2}(X, \nu)$, for some $G$-invariant measure $\nu$ with multiplicity on $X$. By a partition of unity argument, one may then embed the representation $\pi$ of $G$ as a subrepresentation of $\infty \lambda$, the direct sum of countably many copies of the left regular representation $\lambda$. If $\xi: G \rightarrow \ell^{2}(\mathbb{N})$ is continuous with compact support, one checks easily that, for every positive-definite function $\psi$ on $G$, one has:

$$
\int_{G}\langle\xi \mid \xi\rangle_{C^{*}} \psi(g) d g \geqslant 0
$$

which proves the announced statement; see [13, Section 2] for details.

\section{REFERENCES}

[1] C. Berg and P.R. Christensen, 'On the relation between amenability of locally compact groups and the norms of convolution operators', Math. Ann. 208 (1974), 148-153.

[2] P. Baum, A. Connes and N. Higson, 'Classifying spaces for proper actions and K-theory of group $C^{*}$-algebras', in $C^{*}$-algebras 1943-1993, a fifty year celebration (Contemporary Mathematics 167, 1994), pp. 241-291.

[3] A. Connes, Noncommutative geometry (Academic Press, San Diego, 1994).

[4] Y. Derriennic and Y. Guivarc'h, 'Théorème de renouvellement pour les groupes non moyennables', C.R. Acad. Sci. Paris 277 (1973), 613-615.

[5] J. Dixmier, $C^{*}$-algebras (North Holland, Amsterdam, New York, 1977).

[6] S.C. Ferry, A. Ranicki and J. Rosenberg, 'A history and survey of the Novikov conjecture', in Novikov conjectures, index theorems and rigidity, London Math. Society Lecture Notes Series 226 (Cambridge University Press, Cambridge, 1995), pp. 7-66.

[7] R. Godement, 'Les fonctions de type positif et la théorie des groupes', Trans. Amer. Math. Soc. 63 (1948), 1-84.

[8] P. Julg, 'Remarks on the Baum-Connes conjecture and Kazhdan's property T', Fields Institute Communciations 13 (1997), 145-153.

[9] H. Kesten, 'Symmetric random walks on groups', Trans. Amer. Math. Soc. 92 (1959), 336-354. 
[10] K. Jensen and K. Thomsen, Elements of KK-theory (Birkhäuser, Boston, Basel, 1991).

[11] R. Palais, 'On the existence of slices for actions of non-compact Lie groups', Ann. of Math. 73 (1961), 295-323.

[12] J-P. Pier, Amenable locally compact groups (Wiley-Interscience, New York, 1984).

[13] A. Valette, 'On the Baum-Connes assembly map for discrete groups', (preprint, 1997).

Institut de Mathématiques

Rue Emile Argand 11

CH-2007 Neuchâtel

Switzerland 